\title{
Fat atrophy at the site of a subdermal contraceptive implant
}

\author{
Ambika Chadha-Gupta, Anthony Moss
}

\begin{abstract}
Background
Progestogen-only implantable contraceptives are an increasingly popular method of contraception used by women worldwide. The implants are inserted subdermally into the arm and slowly release synthetic progesterone into the systemic circulation to prevent pregnancy.

Over the years, in response to well-publicised side effect profiles, there has been an evolution in the composition and insertion methodology of these implants. Prior to their insertion, patients should be counselled regarding associated risks and side effects at both local and systemic levels. Whilst systemic side effects have been extensively researched, we discuss a case of fat atrophy located at the site of an implant, a hitherto unpublicised local side effect.
\end{abstract}

\section{Case report}

A 27-year-old woman presented for removal of her subdermal contraceptive implant, Norplant ${ }^{\circledR}$, due to persistent vaginal bleeding. The implant had been in place for over 5 years and had been placed on the outer aspect of the left upper arm (vaccination area). At this site, however, a contour deformity was noticed, which measured approximately $6 \times 4 \mathrm{~cm}$ (Figure 1) and had an appearance characteristic of fat atrophy. The woman was otherwise fit and well, and she underwent an uncomplicated removal of the implant under local anaesthetic.

\section{Discussion}

The Norplant system was the first contraceptive implant to be approved for commercial use more than 20 years ago and was used by millions of women in over 60 countries. It comprised six silicone capsules that slowly released the progestogen, levonorgestrel. Despite its well-established contraceptive efficacy, Norplant was dogged by much adverse publicity surrounding both its side effect profile and the complexity of insertion and removal procedures. In 1999, after a 'trial by media', Norplant was withdrawn from the UK market. Ultimately, the manufacturers of Norplant ceased its worldwide distribution in July 2002, reportedly because of a limitation in component supplies. However, as Norplant was marketed as having a 5-year expiration date, many women may still have the implant in place.

Norplant was swiftly superseded by several other brands of progestogen-releasing implants such as Jadelle ${ }^{\circledR}$, a two-rod levonorgestrel-releasing implant, and Implanon ${ }^{\circledR}$, a single etonogestrel-releasing contraceptive

J Fam Plann Reprod Health Care 2006; 33(2): 123-124 (Accepted 8 August 2006)

\section{Department of Neurosurgery, St George's Hospital, London, UK \\ Ambika Chadha-Gupta, BMBCh, MRCs, Senior House Officer}

\section{Department of Plastic Surgery, St George's Hospital, London, UK}

Anthony Moss, FRACS (Plast), FRCS, Consultant

Correspondence to: Miss Ambika Chadha-Gupta, Department of Neurosurgery, Atkinson Morley Wing, St George's Hospital Blackshaw Road, Tooting, London SW17 OQT, UK.

E-mail: ambikachadha@doctors.org.uk

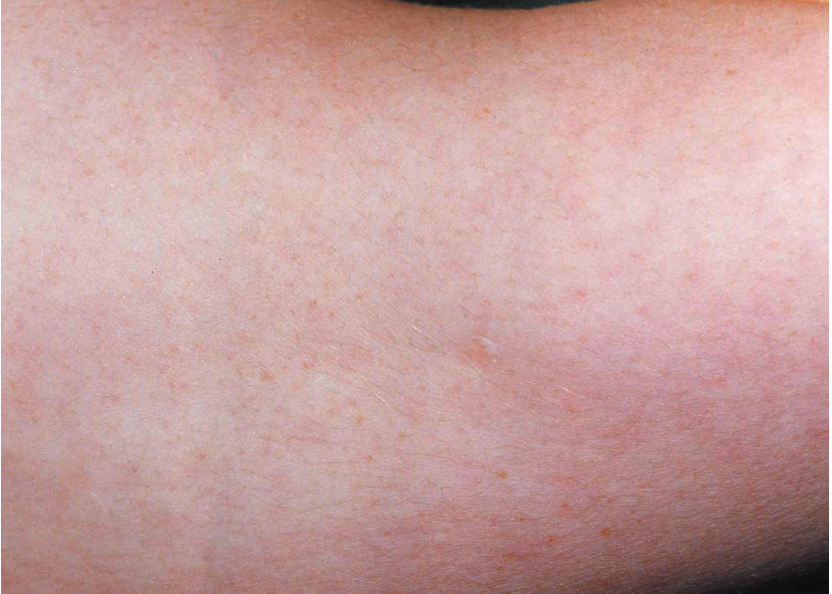

Figure 1 Outer aspect of the patient's left upper arm with a contour deformity characteristic of fat atrophy

implant. This latter system, with a working life of 3 years, is the contraceptive implant currently available to women in the UK, although it is only one of several marketed worldwide.

Common adverse effects of progestogen-only contraceptive implants such as Norplant have been well reported in the literature. They include menstrual disturbances, mastalgia, acne, weight gain and changes in sexual behaviour. ${ }^{1}$ Whilst newer generation implant contraceptives may have improved upon the side effect profile of older ones, the complications and risks of their insertion and removal still remain. Moreover, a formal acknowledgement in the patient literature of side effects local to the insertion site is still pending. Although fat atrophy at the site of Norplant insertion is not a recognised complaint, the loss of contour may have significant aesthetic impact. Furthermore, elucidation of the exact mechanism responsible may predict that it is a side effect common to the newer generation contraceptive implants in use today.

Several categories of progestogens are used in implantable contraceptives including levonorgestrel (Norplant, Jadelle), etonogestrel (Implanon), nestorone (Elcometrine ${ }^{\circledR}$ ) and nomegestrol acetate (Uniplant ${ }^{\circledR}$, Surplant ${ }^{\circledR}$ ). The two main classes of synthetic, nonbiodegradable polymers used to facilitate slow release of these hormones are silicone elastomers (Silastic ${ }^{\circledR}$ ) and ethylene-co-vinyl acetate (EVA; Elvax ${ }^{\circledR}$ ). Fat atrophy local to a subdermal contraceptive may thus be due to any one of the components of such systems that may act directly on surrounding fat cells or indirectly by means of a foreignbody-type reaction.

The use of silicone and its elastomers as a biomaterial is still a source of controversy. Although several studies have established Silastic material as biologically inert, pathological responses and foreign-body reactions to silicone continue to be described with a variable clinical presentation. The exact mechanism of silicone-induced tissue damage is not known, although it may be mediated in part by an immunological reaction. Despite the paucity of literature, the conclusion thus far is that polymers used in implantable contraceptives elicit no local or systemic effects over extended periods in vivo. ${ }^{2}$ 
A more plausible mechanism of fat atrophy is the direct effect of released progestogens on subdermal tissues prior to their circulation in the blood. An analogous means of fat atrophy at the site of intralesional corticosteroids is a wellestablished local side effect of therapy. ${ }^{3}$ Although much research has been conducted on the effects of implantable progestogen-only contraceptives on systemic lipid profiles, the mechanism by which they induce localised fat loss remains unclear. The chemical structure of progestogens and corticosteroids are similar, however, and thus it is possible that they both cause local cutaneous changes by the same process. Although currently undetermined, this process may involve altered expression of adrenoreceptors. ${ }^{4}$ Of relevance are the reported effects of levonorgestrel-releasing intrauterine devices on endometrial glands, causing atrophy of endometrial glands and, on occasion, the whole functional layer. ${ }^{5}$

Fat atrophy at the site of a subdermal contraceptive implant may be specific to the constituents of that particular device. However, the related chemical structure of different progestogen-releasing implants raises the possibility of a similar profile of local side effects common to several brands. Indeed, anecdotal and published reports of contour deformities at the site of dermal implants have been associated with patients who are on their first Norplant device as well as those who have had consecutive but variably branded implants inclusive of Norplant. Atrophic events may therefore be a consequence of time exposure to an implant device in addition to individual sensitivity to implant components.

If fat atrophy is indeed a substantiated local effect of implants then patient counselling should include this information. Furthermore, given that the insertion site of such devices is not standardised, our recommendation is that they are located in the inner aspect of the upper arm so as to minimise the aesthetic impact of any potential fat loss. For those patients wishing to renew an existing implant, consideration should be given to embedding the next implant into that arm that has hitherto been implant-free. Notwithstanding these recommendations, however, it is clear that pharmacovigilance, particularly with respect to local effects of contraceptive implants, is required.

\section{Acknowledgement}

This case report is published with the kind permission of the patient concerned.

Statements on funding and competing interests

Funding None identified.

Competing interests None identified.

\section{References}

1 Rehan N, Inayatullah A, Chaudhary I. Norplant: reasons for discontinuation and side-effects. Eur J Contracept Reprod Health Care 2000; 5: 113-118.

2 Shastri PV. Toxicology of polymers for implant contraceptives for women. Contraception 2002; 65: 9-13.

3 Basadonna PT, Rucco V, Gasparini D, Onorato A. Plantar fat pad atrophy after corticosteroid injection for an interdigital neuroma: a case report. Am J Phys Med Rehabil 1999; 78: 283-285.

4 Monjo M, Rodriguez AM, Palou A, Roca P. Direct effects of testosterone, 17 beta-estradiol, and progesterone on adrenergic regulation in cultured brown adipocytes; potential mechanism for gender-dependent thermogenesis. Endocrinology 2003; 144: 4923-4930.

5 Pakarinen PI, Lahteenmaki P, Lehtonen E, Reima I. The ultrastructure of human endometrium is altered by administration of intrauterine levonorgestrel. Hum Reprod 1998; 13: 1846-1853. 